\title{
Extremal Chemical Trees
}

Miranca Fischermann, Ivan Gutman ${ }^{\mathrm{a}}$, Arne Hoffmann ${ }^{\mathrm{b}}$, Dieter Rautenbach,

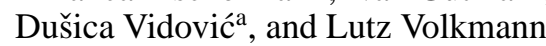

Lehrstuhl II für Mathematik, RWTH-Aachen, D-52056 Aachen

${ }^{a}$ Faculty of Science, University of Kragujevac, P. O. Box 60, YU-34000 Kragujevac

${ }^{\mathrm{b}}$ Lehrstuhl C für Mathematik, RWTH-Aachen, D-52056 Aachen

Reprint requests to Prof. L. V.; Fax: ++49-241-8092136; E-mail: volkm@math2.rwth-aachen.de

Z. Naturforsch. 57 a, 49-52 (2002); received January 14, 2002

A variety of molecular-graph-based structure-descriptors were proposed, in particular the Wiener index $W$, the largest graph eigenvalue $\lambda_{1}$, the connectivity index $\chi$, the graph energy $E$ and the Hosoya index $Z$, capable of measuring the branching of the carbon-atom skeleton of organic compounds, and therefore suitable for describing several of their physico-chemical properties. We now determine the structure of the chemical trees $(=$ the graph representation of acyclic saturated hydrocarbons) that are extremal with respect to $W, \lambda_{1}, E$, and $Z$, whereas the analogous problem for $\chi$ was solved earlier. Among chemical trees with 5, 6, 7, and $3 k+2$ vertices, $k=2,3, \ldots$, one and the same tree has maximum $\lambda_{1}$ and minimum $W, E, Z$. Among chemical trees with $3 k$ and $3 k+1$ vertices, $k=3,4, \ldots$, one tree has minimum $W$ and maximum $\lambda_{1}$ and another minimum $E$ and $Z$.

Key words: Chemical Tree; Branching; Wiener Index; Hosoya Index; Connectivity Index;

Eigenvalue (of a graph); Energy (of a graph).

\section{Introduction}

A general problem of theoretical organic chemistry is to establish the mathematical basis for the connections between molecular structures and physicochemical properties of chemical compounds [1 - 4]. In order to achieve this goal, a remarkably large number of so-called structure-descriptors or "topological indices" has been put forward [5, 6]. Several among them measure the extent of branching of the carbonatom skeleton, a feature related to the molecular surface area [7]. The molecular surface area significantly influences those physico-chemical properties of nonpolar organic molecules (such as alkanes) that are primarily caused by van der Waals intermolecular attraction.

A topological index (TI) acceptable as a measure of branching must satisfy the inequalities

$$
\mathrm{TI}\left(P_{n}\right)>T I\left(X_{n}\right)>T I\left(S_{n}\right), n=5,6, \ldots
$$

or

$$
\mathrm{TI}\left(P_{n}\right)<\mathrm{TI}\left(X_{n}\right)<\mathrm{TI}\left(S_{n}\right), ; n=5,6, \ldots
$$

where $P_{n}$ and $S_{n}$ are the $n$-vertex path graph and star, respectively, and $X_{n}$ is any $n$-vertex tree, different from $P_{n}$ and $S_{n}$. (It is also necessary that small changes in the structure of $X_{n}$ cause small changes in the value of $\operatorname{TI}\left(X_{n}\right)$, a condition which is satisfied by all topological indices considered in this work.) Although no precise definition of "branching" exists, it is obvious that among $n$-vertex trees $P_{n}$ is the least branched and $S_{n}$ the most branched species.

The relations (1) were shown to be obeyed by the Wiener index $W[8]$, the connectivity index $\chi[9,10]$, the graph energy $E$ [11] and the Hosoya index $Z$ [11]; relations (2) are obeyed by the largest graph eigenvalue $\lambda_{1}$ [12].

The Wiener index is defined as $[6,13]$

$$
W=W(G)=\sum_{u<v} d(u, v \mid G),
$$

where $d(u, v \mid G)$ is the distance between the vertices $u$ and $v$ of the molecular graph $G$ and the summation goes over all pairs of vertices. The connectivity index is $[6,14]$

$$
\chi=\chi(G)=\sum_{u, v}\left(\delta_{u} \delta_{v}\right)^{-0.5},
$$

0932-0784 / 02 / 0100-0049 \$ 06.00 @ Verlag der Zeitschrift für Naturforschung, Tübingen · www.znaturforsch.com 
where $\delta_{v}$ denotes the degree (= number of first neighbors) of the vertex $v$, and where the summation extends over all pairs of adjacent vertices of $G$. The Hosoya index is $[6,15]$

$$
Z=Z(G)=\sum_{k \geq 0} m(G, k),
$$

where $m(G, k)$ is the number of $k$-matchings (= selections of $k$ mutually non-touching edges) in $G$. If $\lambda_{j}, j=1,2, \ldots, n$, are the eigenvalues of the graph $G$, then $[11,16,17]$

$$
E=E(G)=\sum_{j=1}^{n}\left|\lambda_{j}\right|
$$

is the energy of the graph $G$. By $\lambda_{1}$ we denote the largest graph eigenvalue, which also has been considered as a measure of molccular branching [18]. Recall that the Hosoya index of trees is directly related to the graph eigenvalues via $[19,20]$

$$
Z=\prod_{j=1}^{n} \sqrt{\left(1+\lambda_{j}^{2}\right)}
$$

Chemical trees are trees (= connected acyclic graphs) in which no vertex has a degree greater than 4 . Such trees provide the natural graph representation of alkanes.

The path graph $P_{n}$ is a chemical tree for all $n=$ $1,2, \ldots$ (representing the normal alkanes). In view of this, the left-hand side of (1) or (2) automatically determines a chemical tree (the least branched one) that is extremal with respect to (w.r.t.) TI. The star $S_{n}$ is a chemical tree only for $n \leq 5$, and therefore the other chemical trees, extremal with respect to TI (the most branched ones), are not determined via (1) or (2). In fact, the finding of these extremal chemical trees showed to be a difficult task.

Some time ago the chemical trees possessing a minimum connectivity index $\chi$ were characterized as follows [21]:

If $n=3 k+2, k=1,2, \ldots$, then the trees consisting only of vertices of degree 1 and 4 have the minimum $\chi$-value among all $n$-vertex chemical trees. If $n=3 k, k=3,4 \ldots$, then minimum $\chi$ is achieved if in addition to vertices of degree 1 and 4 , the tree possesses a single vertex of degree 2 , adjacent to two vertices of degree 4 . If $n=3 k+1, k=4,5, \ldots$, then minimum $\chi$ is achieved if in addition to vertices of degree 1 and 4 , the tree has a single vertex of degree 3 , adjacent to three vertices of degree 4 .

In spite of some earlier work [22], the structure of the chemical trees with minimum Wiener index has not been established. Recently, some of the present authors succeeded in determining the trees with bounded vertex degrees, having minimum $W$-values in [23]; a more general result along these lines was also obtained [24]. By means of the results [23], the chemical trees extremal w. r. t. the Wiener index can now be completely characterized. The structure of these extremal chemical trecs is described in the subsequent section. By numerical testing we found that the very same trees have also maximum $\lambda_{1}$-values and, for certain values of $n$, also minimum $E$ - and $Z$-values.

\section{Chemical Trees with Minimum Wiener Index}

Denote by $\mathcal{T}(n, \Delta)$ the set of trees of order $n$, no vertex of which has a degree greater than $\Delta$. If $\Delta=1$, then $\mathcal{T}(2, \Delta)=\left\{P_{2}\right\}$ and $\mathcal{T}(n, \Delta)=\emptyset$ for $n>2$. If $\Delta=2$, then $\mathcal{T}(n, \Delta)=\left\{P_{n}\right\}$ for $n \geq 2$. These cases are of no interest for our considerations.

Assuming that $\Delta \geq 3$ and $n \geq \Delta+1$, construct a tree $T_{n}(\Delta) \in \mathcal{T}(n, \Delta)$ in the following manner (cf. Fig. 1):

Define auxiliary numbers $M_{0}=1, M_{1}=1+\Delta$ and $M_{j}=1+\Delta+\Delta(\Delta-1)+\Delta(\Delta-1)^{2}+\cdots+\Delta(\Delta-1)^{j-1}$ for $j \geq 2$. Choose $k$ so that $M_{k} \leq n<M_{k+1}$. Then there are unique integers $r$ and $s$, such that

$$
n-M_{k}=r(\Delta-1)+s ; 0 \leq s<\Delta-1 \text {. }
$$
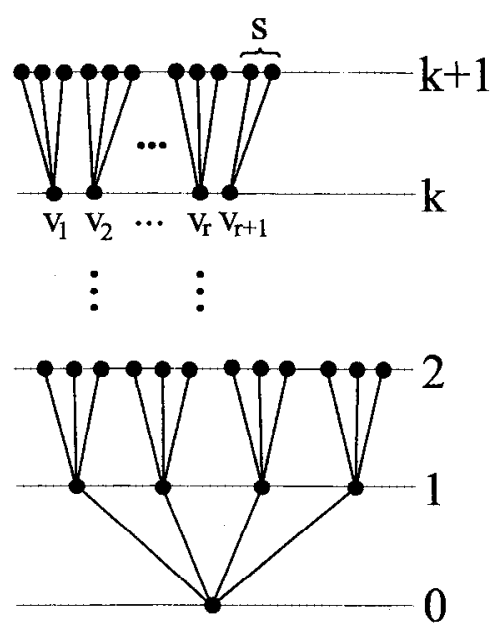

Fig. 1. The structure of the tree $T_{n}(\Delta) \in \mathcal{T}(n, \Delta)$ for $\Delta=4$; for details see text. 


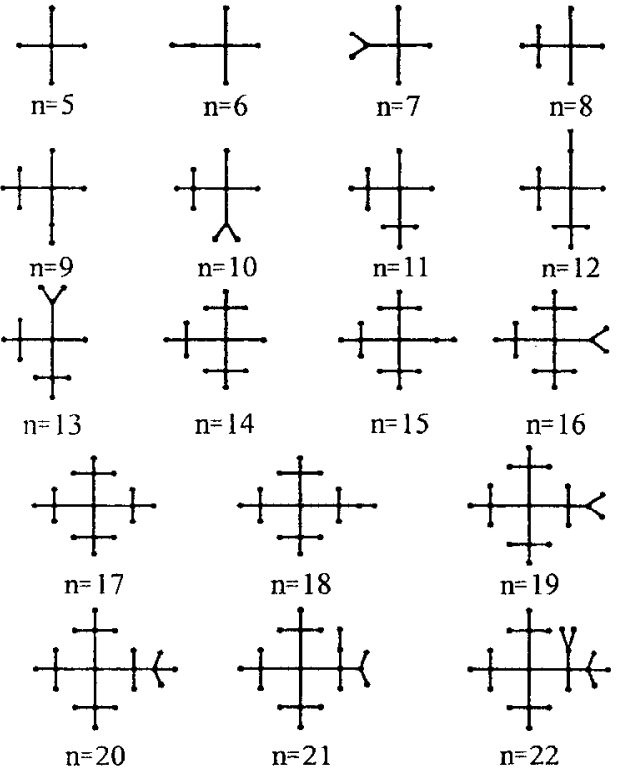

Fig. 2. The chemical trees of order $n, n=5,6, \ldots, 22$, with minimum Wiener index; the same trees have also maximum largest eigenvalue. For $n=6,7$, and $n=3 k+2, k=$ $1,2, \ldots$, these trees have also a minimal connectivity index, Hosoya index and energy.

Arrange the vertices of $T_{n}(\Delta)$ in $k+2$ horizontal rows, so that in the zeroth row there is one vertex, in the $j$-th row, $j=1,2, \ldots, k$, there are $\Delta(\Delta-1)^{j-1}$ vertices, and in the $(k+1)$-th row there are $r(\Delta-$ $1)+s$ vertices. (The $(k+1)$-th row may be empty, if $r=s=0$.)

For $j=1,2, \ldots, k-1$, every vertex in the $j$-th row has a unique neighbor in the $(j \ldots 1)$-th row, and $\Delta-1$ neighbors in the $(j+1)$-th row. If $v_{1}, v_{2}, \ldots, v_{r+1}$ are the $r+1$ leftmost vertices in the $k$-th row such that $v_{i}$ lies on the left of $v_{j}$ for $i<j$, then each of $v_{1}, v_{2}, \ldots, v_{r}$ has $\Delta-1$ neighbors in the $(k+1)$ th row, and $v_{r+1}$ has $s$ neighbors in the $(k+1)$-th row. Consequently, either all vertices of $T_{n}(\Delta)$ are of degree 1 and $\Delta$, or all but one vertices are of degree 1 and $\Delta$ and one vertex (lying in the $k$-th row) has degree $s+1$

In the work [23] it was proven that among the elements of the set $\mathcal{T}(n, \Delta)$, the tree $T_{n}(\Delta)$ has the minimal Wiener index. The special case of this result for $\Delta=4$ provides the chemical trees with minimal Wiencr index.

Since the above described construction is not easy to follow (cf. Fig. 1), in Fig. 2 we show the first few chemical trees extremal w.r. t. the Wiener index.
We see that the minimum-Wiener-index chemical tree with $n$ vertices (denoted by $T_{n}$ ) can always be obtained by attaching a new vertex $v_{n}$ of degree one to some vertex of the minimum-Wiener-index chemical tree with $n-1$ vertices (denoted by $T_{n-1}$ ). The following rules must be obeyed:

(a) The vertices of $T_{n-1}$ are labeled by $v_{1}, v_{2}, \ldots, v_{n-1}$. We may start with $T_{5}$ (the neopentane graph) in which $v_{1}$ is the vertex of degree four and $v_{2}, v_{3}, v_{4}, v_{5}$ are of degree one.

(b) If $T_{n-1}$ does not possess a vertex of degree 2 or 3 , then $T_{n}$ is obtained from $T_{n-1}$ by attaching the new vertex $v_{n}$ to a vertex of degree one. By this the diameter (= maximum distance between two vertices) of $T_{n}$ will either increase by one or will remain the same as the diameter of $T_{n-1}$.

$\left(b_{1}\right)$ If possible, the transformation $T_{n-1} \rightarrow T_{n}$ is to be done so that the diameter remains the same. If there are several possibilities to add a vertex without changing the diameter, then the new vertex $v_{n}$ must be attached to the vertex of degree one with the smallest labcl.

$\left(b_{2}\right)$ If the increase of the diameter in the transformation $T_{n-1} \rightarrow T_{n}$ cannot be avoided, then - again - the new vertex $v_{n}$ must be attached to the vertex of degree one with the smallest label.

(c) If $T_{n-1}$ possesses a vertex of degree 2 or 3 , then $T_{n}$ is obtained from $T_{n-1}$ by attaching the new vertex $v_{n}$ to this vertex of degree 2 or 3 .

\section{Chemical Trees Extremal with Respect to Other Topological Indices}

In the case of the Wiener index, the extremality of the chemical trees $T_{n}$ was verified in a rigorous mathematical manner [23]. For other topological indices of interest (except for the connectivity index [21]) no such approach is available. In order to partially fill this gap we have performed a systematic computer search for chemical trees with maximal $\lambda_{1}$ and minimal Hosoya index and energy. The results obtained, covering chemical trees up to 22 vertices, are depicted in Figs. 2 and 3.

\section{Discussion and Concluding Remarks}

We found that for all (examined) values of $n$, the largest eigenvalue $\lambda_{\mathrm{I}}$ is maximal just for the trees $T_{n}$, a result that may be not a mere coincidence. Providing 


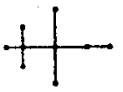

$n=9$
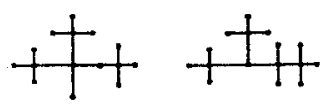

$n=15$

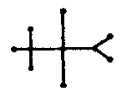

$\mathrm{n}=10$
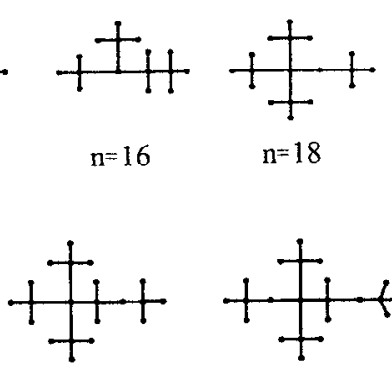

$\mathrm{n}=21$ $n=12$

$n=18$
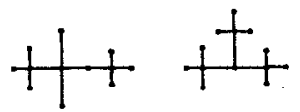

$n=13$

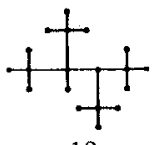

$n=19$
Fig. 3. The chemical trees of order $n=9,10,12,13,15,16$, $18,19,21$, and 22 with minimal Hosoya index and energy. The rule(s) by which these trees are constructed are not easy to envisage.

a proof of this finding may he a feasible challenge for mathematicians.

The same trees $T_{n}$ are, however, not extremal for the connectivity index, except when $n=3 k+2$ (see above).

In the case $n=3 k+2$ the trees $T_{n}$ seem to be extremal also with regard to the Hosoya index $Z$ and

[1] I. Gutman and O. E. Polansky, Mathematical Concepts in Organic Chemistry, Springer-Verlag, Berlin 1986.

[2] R. E. Merrifield and H. E. Simmons, Theor. Chim. Acta 55, 55 (1980).

[3] O. E. Polansky, Z. Naturforsch. 41a, 560 (1986).

[4] M. Zander, Z. Naturforsch. 45a, 1041 (1990).

[5] J. Devillers and A. T. Balaban (Eds.), Topological Indices and Related Descriptors in QSAR and QSPR, Gordon \& Breach, Amsterdam 1999.

[6] R. Todeschini and V. Consonni, Handbook of Molecular Descriptors, Wiley-VCH, Weinheim 2000.

[7] I. Gutman and T. Körtvélyesi, Z. Naturforsch. 50a, 669 (1995).

[8] R. C. Entringer, D. E. Jackson, and D. A. Snyder, Czech. Math. J. 26, 283 (1976)

[9] B. Bollobás and P. Erdös, Ars Combinatoria 50, 225 (1998).

[10] G. Caporossi, I. Gutman, and P. Hansen, Comput. Chem. 23, 469 (1999).

[11] I. Gutman, Theor. Chim. Acta 45, 79 (1977).

[12] L. Lovász and J. Pelikán, Period. Math. Hung. 3, 175 (1973).

[13] II. Wiener, J. Amer. Chem. Soc. 69, 17 (1947). graph energy $E$. Recall that if $n=3 k+2$, then the tree $T_{n}$ possesses only vertices of degree 1 and 4 or, in Cayley's terminology, it is a plerogram [25].

If the number of vertices is such that there necessarily must exist vertices of degree 2 and / or 3 , then the situation becomes less transparent. In Fig. 3 are depicted the chemical graphs with $3 k$ and $3 k+1$ vertices, up to 22 vertices, found to be extremal w. r. t. $Z$ and $E$. These data, however, are insufficient to enable the formulation of a sound conjecture about the structure of such trees for $n>22$. It is likely, but awaits a proof, that for all values of $n$, the trees extremal w. r.t. $Z$ and $E$ coincide.

The extremal trees considered in this work correspond to the alkanes with the most branched carbon-atom skeleton. Because of steric repulsion between the near-lying hydrogen atoms, some of these molecules may possess low chemical stability or even be impossible to exist. On the other hand, if the respective highly branched alkanes could be prepared, then their physico-chemical behavior should be nonstandard. As shown in [7], the molecules of such compounds will have extremely small surface areas, resulting in weak intermolecular attractions. This, in turn, will have various consequences on the physical and chemical behavior of the materials produccd from such compounds [26].

[14] M. Randic, J. Amer. Chem. Soc. 97, 6609 (1975).

[15] H. Hosoya, Bull. Chem. Soc. Japan 44, 2332 (1971).

[16] A. Graovac, I. Gutman, P. E. John, D. Vidović, and I. Vlah, Z. Naturforsch. 56a, 307 (2001).

[17] H. Fripertinger, I. Gutman, A. Kerber, A. Kohnert, and D. Vidović, Z. Naturforsch. 56a, 342 (2001).

[18] D. M. Cvetković and I. Gutman, Croat. Chem. Acta 49, 115 (1977).

[19] I. Gutman and A. Shalabi, Z. Naturforsch. 39a, 797 (1984).

[20] I. Gutman, Z. Marković, and S. Marković, Chem. Phys. Lett. 134, 139 (1987).

[21] I. Gutman, O. Miliković, G. Caporossi, and P. Hansen, Chem. Phys. Lett. 306, 366 (1999).

[22] M. Lepović and I. Gutman, J. Chem. Inf. Comput. Sci. 38, 823 (1998).

[23] M. Fischermann, A. Hoffmann, D. Rautenbach, L. Székely, and L. Volkmann, Discrete Appl. Math., in press.

[24] F. Jelen and E. Triesch, Discrete Appl. Math, in press.

[25] I. Gutman, D. Vidović, and L. Popović, J. Chem. Soc. Faraday Trans. 94, 857 (1998).

[26] D. H. Rouvray, W. Tatong, Z. Naturforsch. 41a, 1238 (1986). 